\title{
然
}

María Marshall*

Carles Domenech**

Carlos Buesa***

\section{LA BIOTECNOLOGÍA EN ESTADOS UNIDOS: OPORTUNIDADES Y CASOS DE ÉXITO}

La biotecnología en Estados Unidos se estructura alrededor de ciudades que aúnan una serie de características que facilitan la formación de empresas biotecnológicas: cercanía a centros de investigación, facilidades burocráticas y presencia de otras biotech. Si bien el sector biotecnológico español es joven en comparación con el americano, la innovación y la buena calidad del sector sanitario en España han hecho que sea capaz de crecer y que algunas empresas hayan podido empezar a instalarse en EE UU con éxito, como es el caso de AbilityPharma y Oryzon.

Palabras clave: patentes, investigación, NIH (National Institute of Health), FDA (Federal Drug Administration), orphan drug, ensayo clínico.

Clasificación JEL: I11, I19.

\section{Panorama general de la biotecnología en EE UU}

Desde el nacimiento de la biotecnología, hace más de medio siglo, Estados Unidos ha mantenido un liderazgo indiscutible; destaca en todas las estadísticas del sector: volumen de ventas, gasto en investigación (I+D), número de empleados, etcétera.

El sector cuenta con una potente estructura empresarial de 2.249 empresas que, en 2018,

\footnotetext{
* Trade Commission of Spain in Chicago.

${ }^{* *}$ CEO, AbilityPharma.

${ }^{* * *}$ CEO, Oryzon.

Versión de febrero de 2019.
}

DOI: https://doi.org/10.32796/bice.2019.3110.6793 generaron unos ingresos de 107.600 millones de dólares y dieron empleo a más de 308.000 personas (IBISWorld, 2018).

El éxito de la industria biotecnológica estadounidense se debe a las sinergias generadas por la combinación de actividad investigadora en el ámbito universitario, el acceso a financiación, la política sanitaria, su sistema de libre mercado y un marco jurídico de protección adecuada de la propiedad intelectual, así como el marco regulatorio estable que proporcionan la FDA (Federal Drug Administration), la EPA (Environmental Protection Agency) y el USDA (United States Department of Agriculture).

En los últimos cinco años se puede notar un primer periodo en el que se produjo una bajada de los ingresos, con motivo de un $D$ 
descenso de la inversión en $\mathrm{I}+\mathrm{D}$, en contraste con una mejora de los ingresos en la segunda mitad de dicho periodo, por el lanzamiento de nuevos productos y otros factores adicionales. Por ejemplo, a medida que nuevos pacientes han contratado seguro médico con motivo de la última reforma sanitaria ha crecido el mercado farmacéutico, que se espera continúe creciendo y, por tanto, lo hagan también los ingresos de la industria biotecnológica.

Uno de los principales motores del sector es el gran número de fusiones y adquisiciones que se dan entre las empresas del mismo. Esto se debe, en parte, al vencimiento de patentes de algunos medicamentos, que provoca que las empresas deseen reforzar su línea de productos con los de las empresas que compran. De esta situación han surgido oportunidades para nuevos agentes en el desarrollo de nuevos fármacos y, como resultado, en los últimos cinco años, el número de empresas de biotecnología ha aumentado a un ritmo anual del 0,8\% (IBISWorld, 2018). Se espera que esta actividad de fusiones y adquisiciones se mantenga elevada en los próximos años y que el mercado se concentre.

Una característica fundamental del mercado estadounidense es que muchas empresas de biotecnología están todavía en fase de desarrollo de productos y no son capaces de generar ingresos a través de las ventas comerciales. En estos casos, las empresas dependen de capital de riesgo o de subvenciones y contratos gubernamentales para continuar con sus investigaciones y ensayos. Por ello, algunas de estas empresas buscan vender sus patentes a las grandes compañías del sector o, incluso, que estas compren la empresa al completo.

Cabe destacar que el número de empresas extranjeras en el mercado es muy reducido y de origen mayoritariamente europeo.
En cuanto al acceso a financiación, se percibe cierta incertidumbre, a pesar de que el sector se ha recuperado de la crisis del 2008. A lo largo de 2017, la financiación con capital riesgo en biotecnología ha disminuido ligeramente respecto a los años anteriores, estableciéndose en 4.413 millones de dólares.

Durante el primer trimestre de 2018, las industrias relacionadas con la biotecnología han recibido una inversión de 1.800 millones de dólares, cifra récord para un solo trimestre, y se han alcanzado 58 acuerdos de colaboración. Sin embargo, se espera que la incertidumbre causada por la inestabilidad del Gobierno provoque una caída de los ingresos a un ritmo anual de $0,7 \%$, alcanzando 103.800 millones de dólares (IBISWorld, 2018).

En cuanto a financiación no dilutiva ${ }^{1}$, las empresas del sector en Estados Unidos pueden acceder a diversas subvenciones: para la investigación, a cargo del NIH (National Institute of Health); para las empresas pequeñas, la SBIR (Small Business Innovation Research); para el desarrollo de nuevas tecnologías, la IRAP (Industrial Research Assistance Program). También pueden recurrir a organizaciones sin ánimo de lucro o fundaciones como Gates, CF, Ellison, Laura \& John Arnold. Para las empresas de biotecnología en fase temprana, expertos del sector recomiendan una combinación de financiación con capital riesgo o de padrinos inversores ${ }^{2}$ con fondos no dilutivos.

En los últimos años se ha visto una subida en los presupuestos del NIH y niveles récord de inversión privada que sin duda ayudan a $\triangleright$

\footnotetext{
1 Financiación no dilutiva: aquella financiación que no provoca la disminución en la propiedad de los accionistas existentes de una empresa (Cinco Días, 2018).

2 Padrino inversor: esta figura no solo invierte su dinero en proyectos de riesgo con la esperanza de obtener retornos cuantiosos e incentivos fiscales, sino que se convierte en el padrino de tales proyectos, esto es, en su valedor, gracias a sus conocimientos en el sector, entre otras aportaciones (Fundéu BBVA, 2018).
} 
contribuir en el avance de la industria y su innovación. No obstante, el mercado no debe ignorar que la competencia internacional en la industria es feroz, y que el ecosistema puede verse afectado por los ciclos económicos, los cambios políticos y las limitaciones presupuestarias.

\section{Oportunidades en el mercado de EE UU}

\subsection{La biotecnología española en EE UU}

La comercialización de la biotecnología está expandiéndose a un ritmo inaudito y con ello surge la necesidad de definir y establecer un compromiso firme con los clientes, inversores y demás agentes del sector. Un buen ejemplo de esto es lo que España ha sido capaz de desarrollar en el sector biotecnológico, aun siendo una industria relativamente joven, en comparación con los líderes mundiales de la industria. En gran medida, esto se debe al carácter transversal que posee la biotecnología y a que cada vez más empresas de diferentes sectores incorporan actividades biotecnológicas a sus productos y servicios permitiendo su crecimiento año tras año.

España ha creado un ecosistema biotecnológico muy dinámico, combinando todos los ingredientes necesarios para fomentar el surgimiento de nuevos proyectos, su desarrollo y crecimiento. El número de publicaciones científicas, las patentes generadas, las empresas semilla o spin-off fundadas, los acuerdos de rondas de financiación y los eventos de liquidez que han tenido lugar en los últimos años son una clara consecuencia de esta realidad.

Si bien el sector español es percibido en EE UU como un mercado pequeño, goza de una imagen de alta calidad investigadora, fundamentalmente en las áreas de oncología, enfermedades neurodegenerativas e inflamatorias, y raras, tanto en investigación básica como en aplicada y en diagnóstico clínico. Las empresas españolas, como parte de la comunidad biotecnológica en EE UU, han podido crear su propia red de contactos y relaciones con otros agentes dentro de la industria.

Las empresas españolas de biotecnología han ido ganando confianza en los últimos años y han pasado a desarrollar actividad comercial e investigadora en EE UU, cuando anteriormente su mercado natural era la Unión Europea. El sector biotecnológico en España crece a un ritmo superior a la media de la UE, convirtiéndose en el quinto país de la Unión Europea en producción científica en biotecnología, por detrás de Reino Unido, Alemania, Francia e Italia.

La interacción entre las empresas españolas y estadounidenses es cada vez mayor, y ha sido objeto de interés de agentes del sector, dando lugar al establecimiento de acuerdos de colaboración tecnológica o comercial, de alianzas y financiación de proyectos.

Una muestra de esto es la implantación de empresas españolas en EE UU, siendo ya 20 los socios de ASEBIO (Asociación Española de Bioempresas) implantados en EE UU (ABTAgarose Bead Technologies, Almirall, Biobide, Bioiberica, Bionure, BTI Biotechnology Institute, Era 7, Esteve, Ferrer, Grifols, Inveready Asset Management, Lipopharma, Natac Biotech, Oryzon Genomics, Pharmamar, Pharmaphenix, Pons IP, RJ Biotech Services, Sanifit y SOM Biotech).

\subsection{El mercado y su distribución}

Las empresas de biotecnología en Estados Unidos están localizadas en diferentes $\triangleright$ 
clústeres, destacando California (San Francisco y San Diego) y Massachusetts (Boston). La posición de liderazgo de estos estados, en particular California, es el resultado de la combinación de la disponibilidad de financiación de capital riesgo, fondos gubernamentales y nivel de regulación en forma de incentivos fiscales; del emprendimiento local y mano de obra cualificada; del prestigio de su red de instituciones académicas, sanitarias y centros de investigación y de la proximidad a grandes empresas de industrias complementarias.
Tal y como se puede ver en el Mapa 1, los cinco estados con más empresas biotecnológicas son: California (19,3\%), Massachusetts (6\%), Nueva York (5,6\%), Florida (5,2\%), Nueva Jersey $(5,1 \%)$ y Texas (5\%). En conjunto, estos cinco estados representan el $40,2 \%$ de los establecimientos relacionados con la biotecnología.

Asimismo, cuando se mide por el número y densidad de establecimientos y empleados, las áreas metropolitanas donde se concentra la actividad del sector son: San Francisco, Boston, San Diego, Los Ángeles, Seattle, $\triangleright$

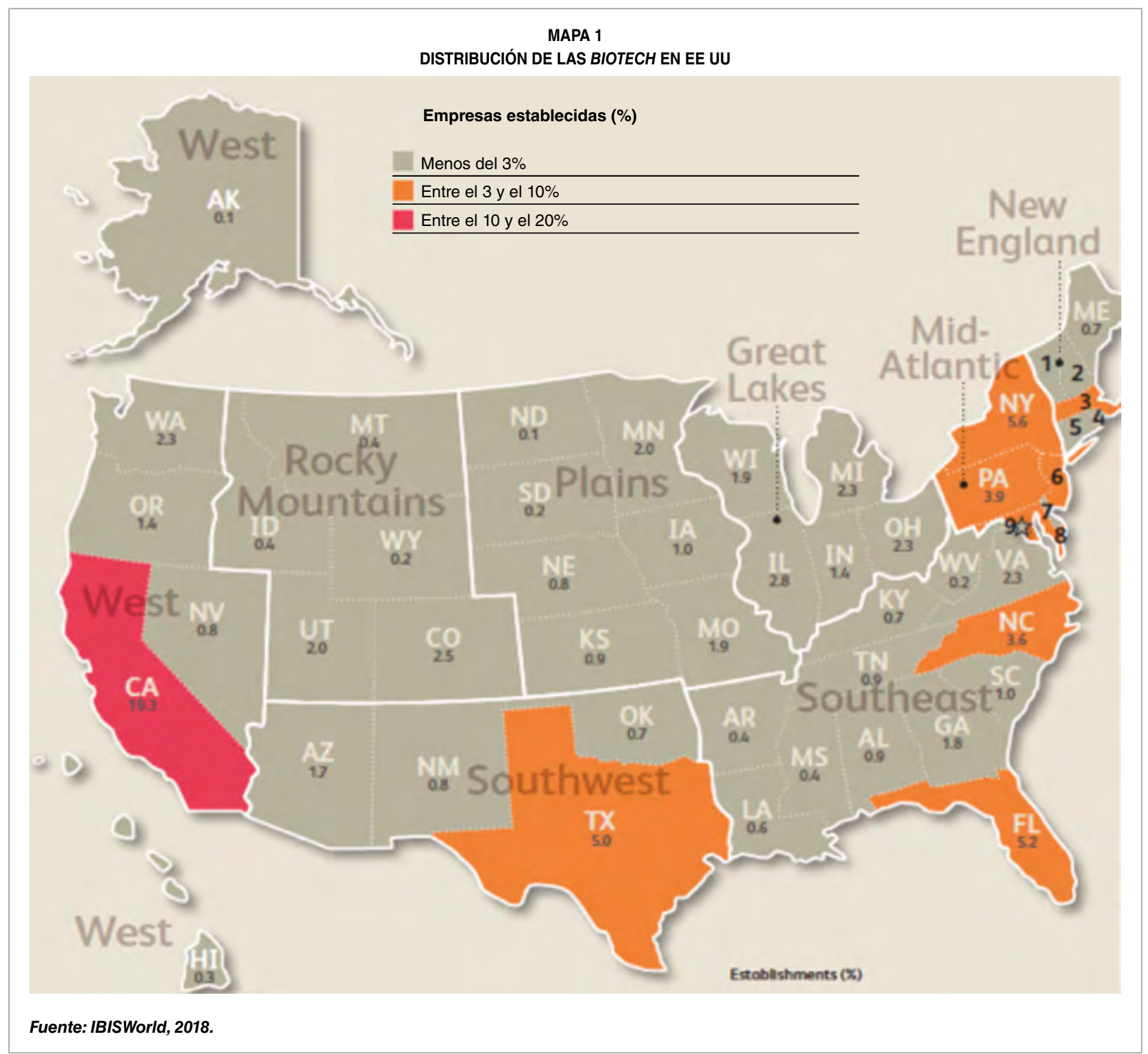


DC, Chicago, Nueva York, Filadelfia y Raleigh (Carolina del Norte).

Cabe destacar el caso de Carolina del Norte, que ha tenido un gran crecimiento en los últimos años, en gran parte debido al llamado triángulo de la investigación, formado por Duke University, North Carolina State University y la University of North Carolina-Chapel Hill.

Los seis factores principales que afectan al desarrollo de la industria biotecnológica en un área geográfica son:

- La disponibilidad de capital riesgo y fondos del Gobierno federal y estatal.

- El nivel de regulación e impuestos del estado.

- Acceso a la investigación a través de universidades y agencias gubernamentales.

- Disponibilidad de personal altamente cualificado.

- Proximidad a grandes empresas privadas en industrias relacionadas.

- Proximidad al área de investigación (es decir, áreas rurales para estudios agrícolas y de océanos para estudios marinos y acuáticos).

De entre los diferentes clusters que cumplen estas características, es necesario resaltar que San Francisco y Boston han acaparado el $50 \%$ de la inversión mundial en biotecnología de fondos de capital riesgo entre 2012 y 2016.

Además, California y Massachusetts son los estados que más financiación reciben del $\mathrm{NIH}$. Cinco de los seis hospitales de investigación independientes financiados por el NIH están en el área de Boston. Estos flujos de fondos contribuyen aún más a la consolidación de la actividad biomédica en estos dos clústeres clave.
El número de estados con un nicho especializado en la industria ha subido de 32 en 2016 a 38 en 2018. Entre estos nichos específicos se encuentran:

- 18 en materias primas agrícolas y en biociencias industriales.

- 11 en la distribución relacionada con la biotecnología.

- 12 en medicamentos y farmacéuticos.

- 14 en dispositivos y equipamiento médico.

- 11 en investigación, ensayos y laboratorios médicos.

Como se puede ver, la industria doméstica de productos biotecnológicos es muy importante, pero el país es deficitario de estos productos, ya que cada vez importa más del extranjero.

Según las perspectivas, proyectadas en la Tabla 1, el mercado americano va a mantener su demanda interna sin grandes variaciones en los próximos años, pero las exportaciones van a seguir subiendo.

Los fabricantes farmacéuticos son el principal cliente de biotecnología en el país, ya que, a menudo, requieren del uso de organismos, procesos $u$ otras tecnologías que han sido desarrolladas por entidades biotecnológicas.

Las compañías farmacéuticas están luchando para desarrollar nuevos productos a medida que se acerca el fin de numerosas patentes. Ya en 2011 expiraron muchas patentes sobre medicamentos de gran éxito, lo que facilitó la entrada al mercado a los fabricantes de genéricos. $\mathrm{Y}$, por ello, muchas compañías farmacéuticas adquirieron medicamentos en última fase de desarrollo de empresas de biotecnología para revitalizar sus propias líneas biotecnológicas. Sin embargo, el proceso de síntesis de células vivas para fármacos biológicos es altamente $D$ 
TABLA 1

CIFRAS DEL SECTOR EN EE UU

(Millones de dólares)

\begin{tabular}{|c|c|c|c|c|}
\hline Año & Ingresos & Exportaciones & Importaciones & Demanda interna \\
\hline 2008 & $89.501,20$ & $16.329,60$ & $23.192,50$ & $96.364,10$ \\
\hline 2009 & $87.957,10$ & $16.349,20$ & $23.658,50$ & $95.266,40$ \\
\hline 2010 & $91.418,70$ & $16.944,10$ & $24.485,20$ & $98.959,80$ \\
\hline 2011 & $95.306,90$ & $17.086,10$ & $25.830,00$ & $104.050,80$ \\
\hline 2012 & $98.668,80$ & $17.456,80$ & $24.473,40$ & $105.685,40$ \\
\hline 2013 & $98.006,00$ & $16.734,50$ & $23.183,50$ & $104.455,00$ \\
\hline 2014 & $98.905,30$ & $17.664,30$ & $24.565,20$ & $105.806,20$ \\
\hline 2015 & $100.945,20$ & $17.602,20$ & $28.593,40$ & $111.936,40$ \\
\hline 2016 & $106.476,70$ & $17.056,10$ & $29.072,00$ & $118.492,60$ \\
\hline 2017 & $106.868,80$ & $16.773,50$ & $28.857,30$ & $118.952,60$ \\
\hline 2018 & $111.076,40$ & $17.273,10$ & $30.041,60$ & $123.844,90$ \\
\hline $2019^{\star}$ & $110.436,50$ & $17.598,00$ & $30.933,40$ & $123.771,90$ \\
\hline $2020^{*}$ & $110.414,20$ & $18.202,90$ & $31.521,80$ & $123.733,10$ \\
\hline $2021^{*}$ & $110.461,50$ & $18.352,60$ & $32.130,80$ & $124.239,70$ \\
\hline $2022^{*}$ & $110.534,50$ & $18.764,30$ & $32.446,30$ & $124.216,50$ \\
\hline
\end{tabular}

variable y propenso a la contaminación, por lo que la producción de biosimilares no supondrá el mismo peligro tras el fin de las patentes.

\section{Casos de éxito}

Para ilustrar el proceso de implantación y desarrollo de negocio de empresas biotecnológicas españolas en el mercado norteamericano, se exponen a continuación los casos de éxito de dos empresas: Ability Pharmaceuticals y Oryzon.

\subsection{Ability Pharmaceuticals inicia su actividad en Estados Unidos}

Ability Pharmaceuticals es una compañía biofarmacéutica comprometida con aumentar la supervivencia de pacientes con cáncer avanzado mediante el desarrollo de medicamentos altamente innovadores que causan la eliminación de las células tumorales a través de autofagia (autodigestión).

La empresa, con sede en Cerdanyola del Vallés (Barcelona), fue creada en 2010, al licenciar la patente de una spin-off de la Universitat de les Illes Balears. Después de una pequeña inversión inicial para realizar las pruebas de concepto en modelos preclínicos, se decidió iniciar el desarrollo del producto ABTL0812, un compuesto anticancerígeno oral innovador, first-in-class, que en pocos años ha llegado a estudios encaminados a la prueba de concepto clínica en personas.

En 2014 la Agencia Española de Medicamentos y Productos Sanitarios (AEMPS) aprobó el inició del primer estudio clínico en humanos, de fase 1/1b, con ABTL0812 en monoterapia, con el que se obtuvieron excelentes resultados de seguridad y signos iniciales de eficacia.

En 2015 AbilityPharma solicitó a la FDA la calificación de medicamento huérfano u ODD $\triangleright$ 
(Orphan Drug Desigantion) para ABTL0812, para el tratamiento del cáncer pediátrico neuroblastoma, que fue aprobada en agosto de 2015, suponiendo la entrada de la empresa en EE UU.

Esta calificación permite a la empresa gozar de un periodo de siete años de comercialización exclusiva en EE UU, así como ciertas facilidades financieras y burocráticas.

Poco después, a finales de 2016, la AEMPS y la Agence Nationale de Sécurité du Médicament et des Produits de Santé (ANSM), en Francia, aprobaron el inicio del ensayo clínico de fase 1/2a (80 pacientes) con ABTL0812 como terapia de primera línea en cáncer de endometrio y en escamoso de pulmón en combinación con paclitaxel y carboplatino. Después de los ciclos de quimioterapia, los pacientes quedan tratados con ABTL0812 de forma crónica. El estudio aún está en curso e incluye centros de primera línea europeos como Vall d'Hebron Institut d'Oncologia VHIO de Barcelona, Institut Gustave Roussy de París, Institut Català d'Oncologia (ICO), Centre Léon Bérard de Lyon, Hospital Clínico de Valencia, Institut PaoliCalmettes de Marsella y Hospital Virgen del Rocío de Sevilla.

En noviembre de 2016, AbilityPharma obtuvo una segunda ODD en EE UU para el tratamiento del cáncer pancreático, y así pudo acceder a financiación pública destinada al desarrollo de medicamentos para enfermedades raras.

En noviembre de 2017 la compañía presentó a la FDA de EE UU la solicitud de ensayos clínicos o IND (Investigational New Drug) con el mismo protocolo para cáncer de endometrio y pulmón actualmente en curso en España y Francia. Un mes después, la solicitud fue aprobada, siendo de las primeras pequeñas biotech españolas en conseguir tener un IND abierto en EE UU y validando así la estrategia clínica y regulatoria en curso en Europa.
A la apertura del IND, en enero de 2018, le siguió una adenda del protocolo para incluir un estudio de ABTL0812 para el cáncer de páncreas en combinación con nab-paclitaxel y gemcitabina en primera línea. Siguiendo esa aprobación, AbilityPharma solicitó la beca Orphan Product Development Grant (R01) de la FDA, que le permitiría subvencionar parte del estudio clínico, y cuya resolución aún está pendiente.

Recientemente, en septiembre de 2018, AbilityPharma obtuvo la aprobación de la ODD para tumores biliares y, actualmente, la empresa quiere seguir expandiéndose en EE UU buscando financiación en ese territorio, ya sea pública o privada.

\subsection{Oryzon: reforzando su presencia en Estados Unidos}

Oryzon Genomics SA es una empresa biofarmacéutica española que representa un claro ejemplo de crecimiento e innovación radical. Surgida en 2000 como una de las primeras spin-off de la Universidad de Barcelona, apostó desde el inicio por la innovación radical, con su plataforma genómica primero y con su posterior conversión en una biofarmacéutica especializada en epigenética que desarrolla sus propios fármacos experimentales epigenéticos para el tratamiento de enfermedades como el cáncer y las enfermedades neurodegenerativas. Su modelo de negocio se basa en avanzar sus fármacos hasta fases clínicas intermedias y después vender la licencia a un socio farmacéutico.

Oryzon es uno de los líderes mundiales en epigenética, con una plataforma epigenética íntegramente desarrollada en sus laboratorios y un extensísimo portfolio (casi una treintena de familias de patentes que se desdoblan en $\triangleright$ 
casi 300 patentes nacionales) que lo convierte en una de las compañías con una posición de propiedad industrial más sólida del sector. La epigenética es un sofisticado mecanismo regulador que permite a las células activar selectiva y reversiblemente la expresión diferencial de determinadas áreas de sus cromosomas y de los genes que allí se encuentran. Ahora se sabe que el inicio y la progresión de algunas enfermedades se deben a una desregulación epigenética, a menudo junto con alteraciones genéticas. El cáncer y los trastornos neurodegenerativos son un ejemplo.

La compañía tiene hoy dos programas terapéuticos en desarrollo clínico, concretamente en fase II, ambos con múltiples oportunidades de indicación: en leucemia mieloide aguda (LMA) y cáncer de pulmón de célula pequeña en el caso de iadademstat, su primera molécula en ser llevada a ensayos clínicos; y en alzhéimer, esclerosis múltiple y agresividad en el caso de vafidemstat, la segunda molécula en desarrollo en sus laboratorios.

Oryzon pasó de ser un spin-off universitario a cotizar en bolsa, y por ello es un ejemplo claro del éxito de las empresas españolas. La salida al mercado, en diciembre de 2015, fue después de una ampliación de capital de 16,5 millones de euros complementada con financiación no dilutiva de más de 15,5 millones de euros adicionales en los meses inmediatamente siguientes. Oryzon se convertía así en la primera compañía puramente biotecnológica en cotizar en el mercado continuo en España.

Oryzon, como todas las compañías europeas en este sector, se plantea cómo financiar su crecimiento y el desarrollo de sus fármacos; una posibilidad es salir a cotizar en el mercado de valores norteamericano: el NASDAQ. La razón es que en el mercado norteamericano se ha desarrollado el ecosistema completo para apoyar una industria tan especial como la industria biotecnológica; desde los fondos de inversión especializados hasta los fondos generalistas y mutuales con presencia importante y continua en el sector; desde los analistas y los banqueros de la banca de inversión hasta los despachos legales especializados en la operativa y las herramientas de inversión de la industria, siguiendo por toda una constelación de profesionales que hacen que el sector pueda financiar sus proyectos de desarrollo de nuevos fármacos y de crecimiento. Inversores sofisticados y habituados a la complejidad científica y al riesgo financiero, que son capaces de apoyar el crecimiento de estas compañías y el desarrollo de sus pipelines.

Oryzon lleva cuatro años con una presencia constante en EE UU, asistiendo a múltiples conferencias científicas o sectoriales organizadas por los diferentes bancos de inversión; reuniéndose con más de 1.500 inversores, abogados, banqueros y consultores de diverso tipo. La compañía tiene desde hace años una oficina en Cambridge (Massachusetts), el mayor hub mundial de la biotecnología.

Sin embargo, no ser una compañía nacida en uno de los campus de excelencia americanos supone que se ha de hacer un esfuerzo extra para llegar a los interlocutores apropiados y poder hacer una presentación adecuada del proyecto y su potencial. La compañía se apoya en su labor en EE UU en firmas de investor relations que ayudan a contactar a inversores y stakeholders importantes para el negocio. Oryzon ha pasado en estos cuatro años, con esfuerzo y perseverancia, de ser un perfecto desconocido en Wall Street a ser un actor más, con un grado de conocimiento razonable, que pugna cada año por su «cuota de visibilidad» con otras farmacéuticas biotech de origen americano. 
Fruto de este trabajo, la compañía se financió utilizando una colocación privada (pipe) en 2017 por 18,2 millones de euros y en 2018 con otro pipe por 13 millones de euros, operaciones lideradas por bancos de inversión norteamericanos y donde fondos norteamericanos especializados y generalistas, pero activos en el sector, obtuvieron por primera vez participaciones relevantes en la compañía.

Oryzon se plantea cotizar en el NASDAQ en un futuro, ya que le permitiría acceder a la plataforma de financiación necesaria para desarrollar moléculas, primeras en su género, que curen o palíen enfermedades muy graves. Una compañía que tenga tal aspiración ha de tener ciertos requisitos: un pipeline con varias moléculas lo suficientemente avanzadas y novedosas en su desarrollo para generar interés entre los inversores, un nivel de madurez en la gestión de la compañía y un conjunto de ejecutivos con experiencia probada en el sector que sepan llevar adelante los ensayos clínicos y garantizar la financiación de la compañía. Y, de no ser norteamericana, una presencia constante en el país.

Oryzon ha reforzado recientemente su presencia en EE UU con la incorporación de un vicepresidente de Desarrollo Clínico con base en San Diego.

\section{Conclusiones}

Estados Unidos es el mercado de biotecnología más grande y dinámico del mundo y, por lo tanto, se trata de un país que las empresas españolas tienen que seguir considerando un objetivo de interés estratégico que les permita aprovechar las indudables ventajas de todo orden que ofrece.
La emergente y dinámica biotecnología española, con bioclústeres que actúan como catalizadores en la construcción del ecosistema de salud y ciencias biológicas de sus respectivas regiones, sigue creciendo año a año y ha conseguido ser reconocida a nivel global. Uno de los principales retos que afronta en este momento es el de introducirse en este atractivo aunque complejo mercado norteamericano, no exento de dificultades, pero que también ofrece interesantes oportunidades como la disponibilidad de capital riesgo, de fondos federales y estatales, de la proximidad a grandes empresas en muchas industrias relacionadas con las diferentes áreas de la biotecnología, etcétera.

Los dos ejemplos analizados, uno de experiencia reciente que nos ofrece Ability Pharmaceuticals y otro de trayectoria consolidada como el de Oryzon, constituyen una buena muestra de las oportunidades que ofrece este mercado para empresas de biotecnología y de la enorme importancia de seguir apostando por el mismo.

\section{Bibliografía}

[1] ASEBIO (2018). Spanish Biotech Industry: Main Success Stories. Recuperado de http:// www.asebio.com/es/documents/ASEBIOcasos deexito.pdf

[2] Curran, J. (2018, diciembre). Gene therapy: More than half of industry revenue is generated by sales of healthcare products. IBISWORLD.

[3] Naval, J. (2018). Barcelona: State-Of-The-Art In Science And Innovation (with a closer look at Life Sciences.

[4] TEConomy/BIO Investment (2018). Investment, Innovation and Job Creation in a Growing U.S. Bioscience. Industry. Recuperado de https://www.bio.org/sites/default/files/ TEConomy_BIO_2018_Report.pdf 